Bei einer auf solchen politisch-kulturellen Tiefenschichten fußenden Parlaments- und Abgeordnetenwahrnehmung muss es aber nicht wundern, wenn die Deutschen wenig Vertrauen zu ihren Abgeordneten haben, ihnen ihr Einkommen missgönnen und gern ignorieren, was die Parlamentarismusforschung und das auf ihr fußende „Leitbild“ der Expertenkommission ihnen über Abgeordnete und ihren Beruf nahebringen wollen. Einmal mehr legt das die Einsicht nahe, dass nicht das politisches System und auch nicht die Politiker der Schwachpunkt unserer Demokratie sind, sondern populäre Missverständnisse und Vorurteile, die das parlamentarische Regierungssystem samt den Abgeordneten recht anders erscheinen lassen, als es tatsächlich ist. Eben das kam - einmal mehr - recht unverfälscht in der populären Kritik an den Empfehlungen der „Diätenkommission“ zum Ausdruck. Also sollte man diese Kritik sehr wohl beherzigen - nur ziemlich anders, als das viele der Kritiker im Sinn hatten.

\title{
Funktionszulagen für Parlamentarische Geschäftsführer. Zum Urteil des Schleswig-Holsteinischen Landesverfassungsgerichts vom 30. September $2013^{1}$
}

\author{
Siegfried Jutzi
}

\section{Hintergrund}

In seinem so genannten ersten Diätenurteil (1975) leitete das Bundesverfassungsgericht (BVerfG) für den Sachbereich der Wahlen aus dem Gleichheitssatz und „der historischen Entwicklung zum Demokratisch-Egalitären hin, die im Grundgesetz für das Bundestagswahlrecht in Art. 38 Abs. 1 Satz 1 und für das Wahlrecht in den Ländern, Kreisen und Gemeinden in Art. 28 Abs. 1 Satz 2 ihren verfassungsrechtlich verbindlichen Ausdruck gefunden hat" ${ }^{\text {“2 }}$ ab, „daß jedem Abgeordneten eine gleich hoch bemessene Entschädigung zusteht, unabhängig davon, ob die Inanspruchnahme durch die parlamentarische Tätigkeit größer oder geringer ist, ob der individuelle finanzielle Aufwand oder das berufliche Einkommen verschieden hoch ist "3. Eine Ausnahme von dem solchermaßen formalisierten Gleichheitssatz erkannte das Gericht lediglich für Parlamentspräsidenten und ihre Stellvertreter an, da sie „an der Spitze eines obersten Verfassungsorgans stehen “4. Das Urteil erfuhr von Anfang an, unter anderem wegen seines ,egalitären Rigorismus “" ${ }^{\text {, }}$, deutliche Kritik. ${ }^{6}$

1 Schleswig-Holsteinisches LVerfG, Urteil vom 30. September 2013, Az. LVerfG 13/12.

2 BVerfGE 40, S. 296, S. 317.

3 Ebenda, S. 318.

4 Ebenda.

5 Klaus Stern, Das Staatsrecht der Bundesrepublik Deutschland, Bd. I, München 1984, \$ 24 II 1, S. 1057.

6 Vgl. nur Hans Hugo Klein, in: Theodor Maunz / Günter Dürig (Hrsg.), Grundgesetz, Kommentar, Art. 48 (Stand: 12/2007), Rn. 168; Stefanie Schmahl, Funktionszulagen - ein Verstoß gegen Mandatsfreiheit und Gleichheit der Abgeordneten?, in: AöR 2005, S. 114 - 149, S. 131 ff.; an- 
Das so genannte zweite Diätenurteil (2000) des BVerfG erging in einem Thüringer Landesorganstreitverfahren nach Art. 93 Abs. 1 Nr. 4 GG. Das BVerfG verortete die Zahlung einer Zusatzentschädigung nicht mehr im Mandat der Abgeordneten, „sondern in besonderen Wahl- und Bestellungsakten des Parlaments“7. Die gesetzliche Gewährung von zusätzlichen Entschädigungen mit Einkommenscharakter im Rahmen der Parlamentsautonomie werde jedoch durch Art. 38 Abs. 1 GG eingeschränkt, da die Zulagen die Entscheidungsfreiheit aller Abgeordneten beeinträchtigen könnten, indem diese „das parlamentarische Handeln am Leitbild einer ,Abgeordnetenlaufbahn' und dem Erreichen einer höheren Einkommensstufe "8 ausrichtete.

Anhand dieser „Leitgesichtspunkte” zur Ermittlung der Grenzen der Parlamentsautonomie gelangte das BVerfG (für Thüringen) zu dem Ergebnis, neben den Mitgliedern des Präsidiums dürften nur die Fraktionsvorsitzenden als „Schaltstelle der Macht im Parlament" ${ }^{\text {"9 }}$ eine zusätzliche Vergütung erhalten, nicht aber Stellvertretende Fraktionsvorsitzende, Parlamentarische Geschäftsführer und Ausschussvorsitzende. ${ }^{10}$

Das zweite Diätenurteil des BVerfG ist im wissenschaftlichen Schriftum ebenfalls auf zum Teil heftige Kritik gestoßen. Es erhebe den formalisierten Gleichheitssatz im Parlamentsrecht zum Dogma ${ }^{11}$, und die Gesamtargumentation sei brüchig, weil die „vom BVerfG entwickelten Vorgaben ... gerade das Gegenteil an Ergebnis erwarten (ließen), als im Urteil konkret judiziert" 12 werde. Außerdem wurde kritisiert, die Subsumtion der Lebenswirklichkeit unter die gefundenen Grundsätze überzeuge nicht ${ }^{13}$, sei wenig konsequent, soweit sie bei den zugelassenen Ausnahmen über Ämter für das Parlament als Ganzes $^{14}$ hinausgehe, und bleibe kryptisch bezüglich der Zahlungen von Fraktionen an ihre Mitglieder. ${ }^{15}$

derer Ansicht zum Beispiel Johannes Hellermann, Von einfachen Abgeordneten und besonderen Funktionsträgern im Parlament. Bemerkungen zum Zweiten Diätenurteil des Bundesverfassungsgerichts vom 21. Juli $2000-2$ BvH 3/91ZG 2001, S. 177 ff., S. 189 f.

7 BVerfGE 102, S. 224, S. 237 unter Bezug auf das Sondervotum von Walter Seuffert, in: BVerfGE 40, S. 296, S. 340.

8 BVerfGE 102, S. 224, S. 239.

9 Ebenda, S. 243.

10 Vgl. ebenda, S. 244.

11 Vgl. Gerald Kretschmer, Das Diätenurteil des Bundesverfassungsgerichts (21. Juli 2000): Vom „fehlfinanzierten“ zum „fehlverstandenen“ Parlament?, in: ZParl, 31. Jg. (2000), H. 4, S. 787 797, S. 788 ff.; zustimmend Lars Brocker / Thomas Messer, Funktionszulagen für Abgeordnete und Oppositionszuschläge - Fortentwicklung der verfassungsgerichtlichen Rechtsprechung durch den Bremer Staatsgerichtshof, in: NVwZ 2005, S. 895 - 898, S. 896, Fn. 3.

12 Vgl. Udo Steiner, Zulagen für besondere Fraktionsfunktionen im Verfassungsstreit, in: BayVBl. 2013, S. 389 - 396, S. 392.

13 Vgl. Lars Brocker / Thomas Messer, a.a.O. (Fn. 11), S. 896 f.; Gerald Kretschmer, a.a.O. (Fn. 11), S. 788 ff.; Hans Hugo Klein, a.a.O. (Fn. 6), Rn. 169 f.; Sven Hölscheidt, Funktionszulage für Abgeordnete, in: DVBl. 2000, 1734 - 1742, S. 1739 f.; Siegfried Jutzi, Zur Abgeordnetenentschädigung, in: NJ 2000, S. 590 - 592, S. 591; Stefanie Schmahl, a.a.O. (Fn. 6), S. 135 ff.; Felix Welti, Funktionszulagen im Konflikt mit Freiheit und Gleichheit der Abgeordneten?, in: DÖV 2001, S. 705 - 714, S. 708 ff.; Joachim Linck, in: ders. / Manfred Baldus / Joachim Lindner / Holger Poppenhäger / Mathias Ruffert (Hrsg.), Die Verfassung des Freistaats Thüringen, Kommentar, Baden-Baden 2013, Art. 54, Rn. 15. Vgl. schon Joachim Linck, Zur Zulässigkeit parlamentarischer Funktionszulagen, in: ZParl, 7. Jg. (1976), H. 1, S. 54 - 60, S. 57 ff.

14 So noch BVerfGE 40, S. 296, S. 318 (nur Präsident und Vizepräsidenten).

15 Vgl. Siegfried Jutzi, a.a.O. (Fn. 13), S. 591. 
In der parlamentarischen Praxis wurde (deswegen?) den Schlussfolgerungen des zweiten Diätenurteils in Bund und Ländern weithin nicht gefolgt. ${ }^{16}$ Auch Verfassungsgerichte der Länder haben Wege gefunden, die Eigenständigkeit ihres Verfassungsraums gegenüber der Rechtsprechung des BVerfG zu verteidigen. ${ }^{17}$

In zwei weiteren Entscheidungen (2007) hat das BVerfG einerseits erneut darauf hingewiesen, „die systematische Ausdehnung von Funktionszulagen“ könne vor Art. 38 Abs. 1 GG keinen Bestand haben ${ }^{18}$, andererseits jedoch betont, dass die „wesentlichen verfassungsrechtlichen Maßstäbe geklärt“ seien, anhand derer die Frage, „für welche Ämter Funktionszulagen vorgesehen werden können, ohne dass die Freiheit des Mandats und der Grundsatz der Gleichbehandlung der Abgeordneten verletzt sind" ${ }^{19}$, beantwortet werden könnte.

\section{Wesentlicher Inhalt des Urteils des LVerfG}

Abgeordnete in Schleswig-Holstein erhalten nach $\$ 6$ Abs. 2 AbgG ${ }^{20}$ für die Ausübung besonderer parlamentarischer Funktionen eine zusätzliche Entschädigung. Sie beträgt für den Landtagspräsidenten 72 v.H., für maximal zwei Vizepräsidenten 13 v.H., für je einen Fraktionsvorsitzenden einer Fraktion 72 v.H., für einen Abgeordneten der dänischen Minderheit, wenn die Stärke einer Fraktion nicht erreicht wird, 45 v.H. und für je einen Parlamentarischen Geschäftsführer pro Fraktion 45 v.H. der monatlichen Entschädigung nach $\$ 6$ Abs. 1 AbgG; Abs. 6 AbgG schließt über die genannten zusätzlichen Entschädigungen hinausgehende Zahlungen für besondere parlamentarische Funktionen aus Mitteln der Fraktionen ausdrücklich aus.

Abgeordnete der Piratenfraktion, die nicht durch Funktionszulagen begünstigt wurden, sahen sich wegen der Zulagen für Parlamentarische Geschäftsführer in ihrem Anspruch auf - auch finanzielle - Gleichbehandlung aller Abgeordneten und auf freie Mandatsausübung (Verstoß gegen Art. 11 Abs. 1 Satz 2 LV SchlH - LV - i.V.m. Art. 38 Abs. 1, Art. 28 Abs. 1 Satz 1 und 2 GG) verletzt. Das hiergegen angestrengte Organstreitverfahren blieb ohne Erfolg.

Das LVerfG, dem als maßgeblicher Prüfungsmaßstab in erster Linie die Landesverfassung zur Verfügung steht, sieht die Ausgestaltung der Abgeordnetenentschädigung (Art. 11 Abs. 1 und 3 LV) als Teil des Statusrechts der Abgeordneten - wie auch das BVerfG - in unmittelbarem Zusammenhang mit den Wahlgrundsätzen des Art. 3 Abs. 1 LV; diese stimmen mit denjenigen, die nach Art. 38 Abs. 1 Satz 1 GG für die Wahlen zum Bundestag gelten, überein und der Landesverfassungsgeber ist zu ihrer Übernahme im Rahmen des Homogenitätsgebots gemäß Art. 28 Abs. 1 Satz 2 GG verpflichtet. ${ }^{21}$

16 Vgl. Stefanie Schmahl, a.a.O. (Fn. 6), S. 116; Udo Steiner, a.a.O. (Fn. 12), S. 394; vgl. auch Hans Herbert von Arnim, Zwischenruf Verfassungsbruch, in: ZRP 2011, S. 185 f., der dies als Verfassungsbruch sieht.

17 Vgl. ThürVerfGH, NVwZ-RR 2003, S. 793 ff.; BremStGH, NVwZ 2005, S. 929 ff.; bereits vor dem zweiten Diäten-Urteil HbgVerfG, DÖV 1997, S. 911 ff.

18 BVerfGE 118, S. 277, S. 329.

19 BVerfGE 119, S. 302, S. 309.

20 Schleswig-Holsteinisches Abgeordnetengesetz - AbgG -, in der Fassung der Bekanntmachung vom 13. Februar 1991, GVOBl. 1991, S. 100, berichtigt GVOBl. 1992, S. 225, zuletzt geändert durch Gesetz vom 16. November 2012, GVOBl. 2012, S. 712.

21 LVerfG, a.a.O. (Fn. 1), Rn. 45 f. 
Das LVerfG betont jedoch die Verfassungsautonomie des Landes. Da sich der Landesverfassung von diesen allgemeinen Anforderungen abgesehen keine konkreten Vorgaben hinsichtlich der Ausgestaltung des Abgeordnetenstatus entnehmen ließen, habe „das Parlament in autonomer Entscheidung unter Abwägung mit den übrigen Strukturentscheidungen zur Ausgestaltung des Parlamentarismus im Land“ die Konkretisierung vorzunehmen. ${ }^{22}$

$\mathrm{Zu}$ diesen im zu entscheidenden Fall relevanten Strukturentscheidungen der Landesverfassung zählt das LVerfG die Hervorhebung der besonderen Bedeutung der Fraktionen für den politischen Meinungsbildungsprozess, die besonders deutlich in Art. $12 \mathrm{LV}$, der die parlamentarische Opposition betrifft, zutage trete. ${ }^{23}$ Daneben spielten die Zusammensetzung (in der Regel 69 Abgeordnete, die nach den Grundsätzen der personalisierten Verhältniswahl gewählt werden) und die Arbeitsweise des Landtages (Vollzeitparlament) eine Rolle. $^{24}$

Ob neben diesen ausdrücklichen Regelungen die aus Art. 38 Abs. 1 und Art. 48 Abs. 3 GG abgeleiteten allgemeinen Grundsätze in Schleswig-Holstein mittelbar oder direkt in die Verfassungsordnung des Landes einwirken, lässt das LVerfG offen, da sich aus dem Grundgesetz kein zusätzlicher verfassungsrechtlicher Maßstab ergebe. Insoweit akzeptiert es unter Hinweis auf das so genannte zweite Diätenurteil des BVerfG das allgemeine, aus der Freiheit und Unabhängigkeit der Mandatsausübung abzuleitende Gebot, dass alle Abgeordneten die gleiche Entschädigung erhalten und Funktionszulagen auf zahlenmäßig begrenzte, besonders herausgehobene politisch-parlamentarische Funktionen beschränkt werden müssen; gleichzeitig betont es jedoch, es gehöre „zum Binnenbereich parlamentarischer Organisation, dass sich der Landtag seine Organisationsstrukturen einschließlich besonders zu entschädigender Funktionsstellen schafft“ und er die Ausgestaltung „in weitgehender Freiheit und unter Berücksichtigung ihrer Flexibilität in Anpassung an die jeweilige Verfassungswirklichkeit" vornehmen kann. ${ }^{25}$

Da das aktuell geltende System den Katalog der Funktionen, für die eine zusätzliche Entschädigung als essentiell für den parlamentarischen Betrieb angesehen wird, knapp halte, die Zahl der zusatzvergütungsfähigen Funktionen einer jeden Fraktion gesetzlich auf eine geringe Zahl beschränkt und jegliche Weiterungsmöglichkeit den einzelnen Fraktionen entzogen sei, sieht das Gericht „die Transparenz und die Rechtfertigungspflicht aller Zulagen im gesamtparlamentarischen Prozess sichergestellt ${ }^{\text {"26. }}$.

Sodann legt das LVerfG im Einzelnen dar, dass Parlamentarische Geschäftsführer eine besonders herausgehobene politisch-parlamentarische Funktion erfüllen. Dies scheitere nicht daran, dass sie - im Unterschied zu anderen Zulagenberechtigten - insbesondere in der Landesverfassung weder ausdrücklich erwähnt (wie Landtagspräsident und Vizepräsidenten) oder vorausgesetzt (wie Fraktionsvorsitzende) werden. Aus einer ausdrücklichen Regelung könne nicht ohne weiteres auf die besondere Heraushebung einer politisch-parlamentarischen Funktion (zum Beispiel Schriftführer in $\$ 6$ GOLT) geschlossen werden. Eine solche könne sich auch aus einer gefestigten parlamentarischen Übung im Landtag

22 Ebenda, Rn. 48.

23 Ebenda, Rn. 49.

24 Ebenda, Rn. 50 f.

25 Ebenda, Rn. 52

26 Ebenda, Rn. 58. Ob auch in einem Modell, das Funktionszulagen auf der Grundlage rein fraktionsautonomer Entscheidungen ermöglicht, den Gefahren für Freiheit und Gleichheit der Abgeordneten begegnet werden kann, lässt das LVerfG offen (Rn. 59). 
ergeben. Hierzu hatte sich das Gericht anhand der Materialien der Benda-Kommission, der Darstellungen des Landtages und der Fraktionen sowie des bestehenden innerfraktionellen Rechts (Geschäftsordnungen der Fraktionen) einen Eindruck von der aktuellen Parlamentswirklichkeit verschafft. ${ }^{27}$ Danach führe die Wahrnehmung der Aufgaben eines Parlamentarischen Geschäftsführers im Schleswig-Holsteinischen Landtag „zu einer solch raumgreifenden Beeinträchtigung der Mandatsausübung der jeweiligen Abgeordneten, dass eine Kompensation mittels einer zusätzlichen Vergütung keine ungerechtfertigte Beeinträchtigung des Grundsatzes gleicher Entschädigung darstellt “28. Das Gericht rechtfertigt also die zusätzliche Entschädigung nicht mit der aus der übernommenen Aufgabe einhergehenden zeitlichen Belastung, sondern aus der Beschränkung der grundsätzlichen Freiheit der Mandatsausübung infolge der Übernahme der Funktion. ${ }^{29}$

Abschließend legt das LVerfG dar, warum es nicht gehalten war, das Verfahren auszusetzen und eine Entscheidung des BVerfG nach Art. 100 Abs. 3 GG einzuholen. Dabei lässt es offen, inwieweit den Feststellungen des BVerfG zur damaligen Rechtslage in Thüringen in seinem so genannten zweiten Diätenurteil unter Berücksichtigung von $₫ 31$ Abs. 1 BVerfGG überhaupt bindende Wirkung zukommen könnte, da es seiner Entscheidung Maßstäbe zu Grunde gelegt habe, die denen des BVerfG nicht widersprächen. ${ }^{30}$ Hiervon zu trennen seien die Ausführungen des BVerfG zu dem seinerzeit konkret zu beurteilenden Sachverhalt in Thüringen. ${ }^{31}$

\section{Kritik}

\subsection{Verfassungsautonomie}

Das BVerfG hatte im zweiten Diätenurteil die Gewährung von Funktionszulagen zu Recht der Parlamentsautonomie und der Selbstorganisation überantwortet, im Ergebnis jedoch Parlament und Fraktionen den politischen Spielraum wieder genommen. ${ }^{32}$ Das LVerfG hat die Verfassungs- und Parlamentsautonomie des Landes gegenüber dem BVerfG elegant verteidigt, ohne die direkte Kontroverse zu suchen, indem es die vom BVerfG dem Homogenitätsgebot entnommenen Grundsätze auch in der Landesverfassung verankert sieht ${ }^{33}$, diese - unbeschadet der Frage, ob es eine rechtliche Bindung in dem zu Thüringen entschiedenen Fall geben kann ${ }^{34}$ - konsequent und auf einer breiteren faktischen Entscheidungsgrundlage als die Karlsruher Richter anwendet, jedoch - oder deshalb? - zu einem anderen Ergebnis als diese gelangt.

Diese Linie wird am Ende der Entscheidung beibehalten, indem das LVerfG es ablehnt, eventuelle Zweifel im Verfahren nach Art. 100 Abs. 3 GG klären zu lassen. Auch dies ist Ausdruck eigenstaatlichen Selbstbewusstseins zur Wahrung des eigenen Verfassungsraums.

27 Ebenda, Rn. 62 f.

28 Ebenda, Rn. 66.

29 Ebenda, Rn. 69; dazu Stefanie Schmahl, a.a.O. (Fn. 6), S. 138 ff.

30 Ebenda, Rn. 72 f.

31 Ebenda, Rn. 78 ff.

32 Vgl. Udo Steiner, a.a.O. (Fn. 12), S. 393.

33 Vgl. auch BVerfGE 120, S. 82, S. 102.

34 Dazu ausführlich Udo Steiner, a.a.O. (Fn. 12), S. 391 f. 
Das Vorgehen erinnert etwas an die Handhabung des vom BVerfG beschworenen „Kooperationsverhältnisses“ ${ }^{\text {"35 }}$ zum EuGH. So sah sich das Gericht ${ }^{36}$ kürzlich „gezwungen“, die Rechtsprechung des EuGH ${ }^{37}$ ultra-vires-akt-vermeidend zu interpretieren, um nicht „Schutz und Durchsetzung der mitgliedstaatlichen Grundrechte in einer Weise“ zu gefährden, „dass dies die Identität der durch das Grundgesetz errichteten Verfassungsordnung in Frage stellte" 38 . Allerdings wäre im Bundesstaat des Grundgesetzes dessen Verfassungsordnung gegenüber einem Land bei „Fehlinterpretationen“ leichter durchsetzbar (vgl. nur Art. 28 Abs. 3 GG), als dies im Verhältnis der EU zu einem Mitgliedstaat möglich ist.

\subsection{Funktionszulagen für Parlamentarische Geschäftsführer}

Die konkrete Anwendung der mit dem Grundgesetz übereinstimmenden Beurteilungsmaßstäbe ist dem LVerfG überzeugend gelungen. Die Gefahr einer Fehlinterpretation des BVerfG erscheint eher gering, wenngleich dieses - mag seine Entscheidung auch keine Bindungswirkung für andere Länder entfalten ${ }^{39}$ - die Funktionszulagen für Parlamentarische Geschäftsführer gerade für ein Land vergleichbarer Größe ${ }^{40}$ verworfen hatte.

Das LVerfG kann sich mit gutem Grund auf die vom BVerfG in der Entscheidung zu Thüringen angestellten allgemeinen Erwägungen stützen, etwa „Regelungen über die innere Ordnung des Parlaments bedürfen der Flexibilität, um eine Anpassung an die geänderte Verfassungswirklichkeit zu ermöglichen“ oder „hinsichtlich der Reichweite und Grenzen der Parlamentsautonomie in Bezug auf Fraktionszulagen lassen sich daher nur sehr allgemeine Kriterien aufzeigen, die als Leitpunkte dienen können“ 41.

Ergänzend dazu ist auf die Entscheidung des BVerfG ${ }^{42}$ aus dem Jahr 2007 hinzuweisen, wonach die „wesentlichen verfassungsrechtlichen Maßstäbe geklärt“ seien, anhand derer die Frage, „für welche Ämter Funktionszulagen vorgesehen werden können“, zu beantworten ist. Auch daraus wird deutlich, dass die im Jahr 2000 für Thüringen konkret gezogen Folgerungen nicht in Stein gemeißelt sind. ${ }^{43}$

35 Vgl. BVerfGE 89, S. 155, S. 175; BVerfGE 102, S. 162, S. 163; zur Entwicklung der Rechtsprechung des BVerfG vgl. zum Beispiel Ulrich Fastenrath / Thomas Groh, Europarecht, Stuttgart / München 2012, Rn. 566 ff.

36 BVerfG, NJW 2013, S. 1499 ff.

37 NJW 2013, S. 513 - Akerberg Fransson; dazu Hans-Jürgen Rabe, Grundrechtsbindung der Mitgliedstaaten, in: NJW 2013, S. 1407 ff.; Daniel Thym, Die Reichweite der EU-GrundrechteCharta - Zu viel Grundrechtsschutz?, in: NVwZ 2013, S. 889 - 896; Christoph Ohler, Grundrechtliche Bindungen der Mitgliedstaaten nach Art. 51 GRCh, in: NVwZ 2013, S. 1436 ff.; Wolfgang Weiß, Grundrechtsschutz durch den EuGH: Tendenzen seit Lissabon, in: EuZW 2013, S. $287-292$, S. 288 f.

38 BVerfG, NJW 2013, S. 1499, Rn. 91.

$39 \mathrm{Zu}$ Recht verneinend Stefanie Schmahl, a.a.O. (Fn. 6), S. 118 f.; Udo Steiner, a.a.O. (Fn. 12), S. $391 \mathrm{ff}$.

40 Thüringen hatte im Jahr 2000 circa 2,5 Millionen Einwohner; Schleswig-Holstein hat heute circa 2,8 Millionen Einwohner; vgl. http://www.statistik-portal.de/statistik-portal/de_jb01_jahrtab1. asp (Abruf am 16. Mai 2014).

41 BVerfGE 102, S. 224, S. 240.

42 BVerfGE 119, S. 302, S. 309.

43 Vgl. auch Stefanie Schmahl, a.a.O. (Fn. 6), S. 147; Udo Steiner, a.a.O. (Fn. 12), S. 392; Christoph Krönke, Verfassungsmäßigkeit von Funktionsvergütungen im Deutschen Bundestag, in: DVBl. 2013, S. $1492-1497$, S. 1495. 
Es kommt hinzu, dass das zweite Diätenurteil von der ganz überwiegenden Meinung nicht so sehr wegen der allgemeinen Maßstäbe, sondern vor allem wegen fehlender Konsequenz und realitätsferner Bewertung der Funktionsträger zu Recht kritisiert wird. ${ }^{44}$ Insbesondere unterscheidet das BVerfG nicht mehr zwischen Ämtern, die das Parlament als Ganzes vergibt, sowie Fraktionsämtern und rechnet - in völliger Verkennung der tatsächlichen und rechtlichen Lage - die Schaffung der Funktionsstellen für Fraktionsvorsitzende dem Landtag zu. ${ }^{45}$

Ausgehend von der gewissen Flexibilität, die in der Rechtsprechung des BVerfG (in unterschiedlichen Funktionen) selbst erkennbar geworden ist, und des Umstands, dass die vom Gericht aufgestellten allgemeinen Maßstäbe explizit abwägungs- und damit ergebnisoffen sind, liegt die Annahme nicht fern, dass der Gestaltungsspielraum der Parlamente in Bund und Ländern bei Funktionszulagen nicht auf die im zweiten Diätenurteil für Thüringen damals zugelassenen Ämter beschränkt ist. Zwar dürfte kaum zu erwarten sein, dass sich das BVerfG von „der historischen Entwicklung zum Demokratisch-Egalitären “46 in erheblichem Umfang distanzieren wird, was auch nicht wünschenswert wäre. ${ }^{47}$ Jedoch dürfte die Hoffnung nicht unbegründet sein, dass das Gericht in Zukunft, wie dies das LVerfG erfreulicherweise getan hat, seine Subsumtion realitätsnäher und auf Tatsachen gestützt vornehmen und deshalb zu anderen Ergebnissen gelangen wird. Auch die vom Ältestenrat des Deutschen Bundestags eingesetzte Unabhängige Kommission zu Fragen des Abgeordnetenrechts hält daher „Funktionsvergütungen auch für Ausschussvorsitzende, stellvertretende Fraktionsvorsitzende, Parlamentarische Geschäftsführer und die Vorsitzenden von Arbeitsgruppen/-kreisen für verfassungskonform “48.

Entscheidend werden die Funktionszulagen gewährenden Parlamente darauf zu achten haben, wegen des aus der Freiheit und Unabhängigkeit der Mandatsausübung abgeleiteten Gebots grundsätzlich gleicher Entschädigung der Abgeordneten den Ausnahmecharakter der Funktionszulagen zu wahren. ${ }^{49}$ In diesem Rahmen dürfte den Parlamenten ein gewisser Gestaltungsspielraum zustehen, und zwar sowohl bei der Einstufung, welche Aufgaben mit dem Prädikat einer besonders herausgehobenen politisch-parlamentarischen Funktion versehen werden dürfen, als auch bei der Frage, ob die Finanzierung unmittelbar durch das Parlament oder über die Fraktionen ${ }^{50}$ erfolgen soll. Die Entscheidung muss jedoch in jedem Fall als Ausfluss der Parlamentsautonomie in ihrem Kern dem Parlament als Ganzem zurechenbar bleiben. Daneben spricht einiges dafür, die aus dem Abgeordnetenmandat ab-

44 Vgl. nur Lars Brocker / Thomas Messer, a.a.O. (Fn. 11), S. 897; Siegfried Jutzi, a.a.O. (Fn. 13), S. 591; Hans Hugo Klein, a.a.O. (Fn. 6), Rn. 170; Gerald Kretschmer, a.a.O. (Fn. 11), S. 788 f.; Stefanie Schmahl, a.a.O. (Fn. 6), S. $131 \mathrm{ff}$.

45 BVerfGE 102, S. 224, S. 242; dazu Hans Hugo Klein, a.a.O. (Fn. 6), Rn. 411e; Wolfgang Zeh, Schriftliche Stellungnahme zu Fragen der Unterkommission des Ältestenrates des Landtags Mecklenburg-Vorpommern, Kommissions-Drs. 16/1, S. 9 f.

46 BVerfGE 40, S. 296, S. 317.

47 Vgl. auch Stefanie Schmahl, a.a.O. (Fn. 6), S. $127 \mathrm{ff}$.

48 BT-Drs. 17/12500 vom 19. März 2013; dazu ausführlich Christoph Krönke, a.a.O. (Fn. 43), S. $1495 \mathrm{f}$.

49 Vgl. auch BVerfGE 118, S. 277, S. 329; BVerfGE 119, S. 302, S. 309.

50 Anderer Ansicht Joachim Linck, in: ders. / Manfred Baldus / Joachim Lindner / Holger Poppenhäger / Mathias Ruffert, a.a.O. (Fn. 13), Art. 58, Rn. 32, dessen Bedenken jedoch durch eine hinreichend bestimmte Ermächtigung des Gesamtparlaments zerstreut werden dürften. Zur Praxis in den Ländern vgl. Stefanie Schmahl, a.a.O. (Fn. 6), S. 121 ff. 
zuleitende Fraktionsautonomie ${ }^{51}$ ebenfalls zu respektieren. Dies bedeutet, dass es auch möglich, ja der Fraktionsautonomie am ehesten gerecht wird ${ }^{52}$, wenn die Zulage aus den der Fraktion zugewiesenen Mitteln erfolgt, wobei es dem Parlament obliegt, hinreichend präzise Regelungen ${ }^{53}$ aufzustellen, welche die Zahl der möglichen Ämter eindeutig beschränken, finanzielle Kumulierungseffekte ausschließen ${ }^{54}$ sowie die Transparenz ${ }^{55}$ und eine effektive Kontrolle der Mittelvergabe sicherstellen.

51 Vgl. Hermann Butzer, in: Volker Epping / Christian Hillgruber (Hrsg.), Grundgesetz, Kommentar, München 2013, Art. 38, Rn. 133 ff.; Hans Hugo Klein, in: Theodor Maunz / Günter Dürig (Hrsg.), Grundgesetz, Kommentar, Art. 48 (Stand: Oktober 2010), Rn. 168; vgl. auch Suzanne S. Schüttemeyer, Fraktionen im Deutschen Bundestag 1949 bis 1997. Empirische Befunde und theoretische Folgerungen, Opladen 1998, S. $30 \mathrm{ff}$.

52 Vgl. Lars Brocker, in: Rudolf Dolzer / Karin Graßhof / Wolfgang Kahl / Christian Waldhoff(Hrsg.), Bonner Kommentar zum Grundgesetz, Art. 40 (Stand: Februar 2011), Rn. 188; Lars Brocker 1 Thomas Messer, a.a.O. (Fn. 11), S. 897 f.; vgl. auch Sven Hölscheidt, Funktionszulage für Abgeordnete, in: DVBl. 2000, S. 1737 - 1742, S. 1742.

53 Zur Regelungsform vgl. Hermann Butzer, a.a.O. (Fn. 51), Rn. 137.

$54 \mathrm{Vgl}$. zum Beispiel $\$ 6$ Abs. 5 AbgG: „Nehmen Abgeordnete mehrere besondere parlamentarische Funktionen wahr, steht ihnen nur die jeweils höchste zusätzliche Entschädigung ... zu."

55 Zum Transparenzgebot vgl. LVerfG, a.a.O. (Fn. 1), Rn. 58.

\section{Von der Affäre zur Reform. Wandlungen des Abgeordnetenrechts in Bayern}

Heinrich Oberreuter

Innerhalb eines Jahres sind in Bayern weite, für die Finanzausstattung der Parlamentarier wesentliche Bereiche des Abgeordnetenrechts tiefgreifenden Änderungen unterzogen worden. ${ }^{1}$ Bis dahin hatte das Maximilianeum hinsichtlich geradezu kontrollfreier Modalitäten bei Arbeits-, Dienst- und Werkverträgen zur Unterstützung der parlamentarischen Arbeit sowie äußerst zurückhaltender Anzeigepflichten von Nebentätigkeiten im Vergleich zu anderen Parlamenten in Deutschland eine Sonderstellung eingenommen. Abhilfe schaffen nun zwei Novellierungen des Abgeordnetengesetzes (vom 22. Mai 2013 und 26. März 2014), verschärfte Verhaltensregeln (vom 16. Juli 2013) sowie völlig neue durchgreifende Richtlinien zu Mitarbeiter- und Werkverträgen (vom 18. Juli 2013). Bei diesen Verträgen wurden wesentliche Positionen der schon seit 1999 dokumentierten landtagsinternen Kritik sowie aktuelle Empfehlungen des Bayerischen Obersten Rechnungshofes (ORH) übernommen.

1 Als haltlos erwies sich der mehrfach geäußerte Verdacht, die Reform werde auf den Sankt-Nimmerleins-Tag verschoben, bei Hans-Herbert von Arnim, Abgeordnetenmitarbeiter und Kostenpauschalen in Bayern. Stellungnahme nach Vorlage des Berichts des Bayerischen Obersten Rechnungshofs, in: Neue Zeitschrift für Verwaltungsrecht, 32. Jg. (2013), H. 19, S. 1262 - 1263 (die ausführliche Fassung erschien vorab online in: Neue Zeitschrift für Verwaltungsrecht - Extra, 32. Jg. (2013), H. 19, S. 1 - 13). 